detect bias due to unmeasured confounding; and 3) to combine estimates from population-based and case-control studies in meta- analyses.

Methods This whole-of-population study of children born from 1999-2013 ( $\mathrm{n}=286,058$, aged $<15$ years) used de-identified linked administrative datasets from the South Australian Early Childhood Data Project. T1D was diagnosed for 557 children during hospitalization (ICD-10-AM codes, E10, E101E109) from 2001-2014. Maternal smoking data was sourced from the South Australian Perinatal Statistics Collection, where information at birth is collected by midwives/neonatal nurses using a validated tool. Hospitalization for any injury occurring at school was used as a negative-control outcome. Adjusted Cox proportional hazard ratios (HR) were calculated in the main analysis and the negative-control outcome. Randomeffects meta-analysis was used to summarize effects of prenatal smoking on childhood T1D.

Results Compared with non-smokers, smoking throughout pregnancy was associated with 23\% lower childhood T1D risk (HR 0.77; 95\% CI 0.60-1.00), with similar effects for smoking in first-half (HR 0.78 ; 95\% CI 0.60-1.01) and secondhalf (HR 0.75 ; 95\% CI $0.57-0.98$ ) of pregnancy. The negative-control outcome analysis (HR 0.95 ; 95\% CI $0.86-1.05$ ) suggested the effect of prenatal smoking on T1D was not due to unmeasured confounding. These results were consistent with meta-analytic estimates of prenatal smoking and childhood T1D risk from population-based (HR 0.70; 95\% CI $0.60-0.81$ ) and case-control studies (OR 0.71 ; 95\% CI $0.55-$ $0.86)$.

Conclusion Maternal smoking in pregnancy was associated with lower risk of childhood T1D. The negative-control outcome analysis suggests this effect is unlikely to be due to unmeasured confounding. Our meta-analytic estimates also showed lower risk of T1D for children exposed to maternal smoking during pregnancy. Smoking causes irreversible harm to the fetus, however understanding the mechanism of protective effects (e.g. nicotine exposure, gene expression) might lead to new insights about T1D.

\section{OP75 A SYSTEMATIC REVIEW AND META-ANALYSIS OF RISK FACTORS FOR PREGNANCY-ASSOCIATED STROKE}

${ }^{1} \mathrm{R}$ Green*, ${ }^{1} \mathrm{~L}$ Tata, ${ }^{2} \mathrm{~N}$ Sprigg. ${ }^{1}$ Division of Epidemiology and Public Health, University of Nottingham, Nottingham, UK; ${ }^{2}$ Division of Clinical Neuroscience, University of Nottingham, Nottingham, UK

\subsection{6/jech-2019-SSMabstracts.78}

Background Stroke in younger women is rare, however, pregnant women have a significantly increased risk around delivery and in early postpartum. Despite known risk factors, such as high blood pressure, the contribution of female-specific factors to women's stroke risk are poorly understood. Whilst there are pathophysiological reasons for increased stroke in pregnancy, it is of clinical and public health importance to determine the extent to which these strokes can be pre-identified by background risk and pregnancy-related factors. We conducted a systematic review to identify risk factors for pregnancy-associated stroke; this included risk factors pre-existing (to pregnancy) and those developing during pregnancy and labour.

Methods An electronic search of PubMed, MEDLINE and EMBASE databases, without language, study design or publication date restrictions, was performed in November 2018.
Study inclusion criteria were reported risk factors/characteristics for women with stroke during pregnancy or up to 12 months after delivery and for a comparison group of pregnant/postpartum women without stroke. Stroke timing (antenatal, perinatal, postnatal), diagnostic type and fatality were assessed. Data were extracted and, where possible, a random effects meta-analysis was conducted, heterogeneity quantified using $\mathrm{I}^{2}$. Methodological quality was assessed using an adapted Newcastle-Ottawa scale.

Results Of 3784 papers screened, 9 studies met the inclusion criteria comprising 11,398 women with stroke and $>85$ million comparison women across 4 countries. Eight studies reported effect measures for at least one risk factor. Of fourteen risk factors reported, 8 showed a statistically significant increase in pregnancy-associated stroke; pooled odds ratios with 95\% confidence intervals: maternal age ${ }^{3} ; 35$ years 2.66 (1.83-3.87), black ethnicity $1.56(1.35-1.81)$, smoking 1.96 (1.64-2.34), alcohol use 2.32 (1.41-3.81), drug abuse 1.82 (1.19-2.77), hypertension 4.80 (3.26-7.06), pre-eclampsia $10.30(8.26-12.84)$ and cesarean delivery 4.85 (2.22-10.59). Parity, body mass index, obesity, diabetes, infection, and migraine were not associated. Studies provided limited data to assess risk factors according to stroke timing, type and fatality.

Conclusion Our findings improve current understanding of the relative contributions of different risk factors for pregnancyassociated stroke. However, our work highlights the very few existing studies in this area. The available studies assessed a limited number of risk factors, and many were similar to those known to increase stroke regardless of pregnancy. Studies including detailed risk assessment in relation to pregnancy, delivery and postpartum complications as well as women's background risks are needed. Additionally, future research should establish whether risk factors differ according to stroke type and time-period in relation to pregnancy.

\section{OP76 A SYSTEMATIC REVIEW AND META-ANALYSIS ON NEURODEVELOPMENTAL EFFECTS OF PRENATAL VITAMIN D IN HUMANS}

E Morales*, AM Garcia-Serna. Pediatrics Research Group, IMIB-Arrixaca Biomedical Research Institute, Murcia, Spain

\subsection{6/jech-2019-SSMabstracts.79}

Background Vitamin D plays a key role in brain development and function; however, evidence in humans has never been systematically reviewed. Hence, we conducted a systematic review, accompanied by meta-analyses where possible, to summarize the existing evidence in humans on the relationship between prenatal 25-hydroxyvitamin D [25(OH)D] circulating levels and neurodevelopmental outcomes, including cognition, psychomotor performance, language development, behavior, ADHD, and autistic traits.

Methods PubMed, Web of Science and SCOPUS databases were systematically searched using keywords. Study eligibility criteria were: 1) original epidemiologic study performed in humans; 2) available information on circulating concentration of $25(\mathrm{OH}) \mathrm{D}$ in maternal or newborn blood as exposure; 3 ) outcome assessment included information on the offspring's neurodevelopment evaluated by standardized test scores; and 4) available data on the relevant estimates of effect size and the corresponding 95\% confidence intervals (CIs). For all the 
analyses the lowest category of $25(\mathrm{OH}) \mathrm{D}$ concentration was defined as reference. Heterogeneity among studies was evaluated by using the Cochran $\mathrm{Q}$ test and quantified by using the $\mathrm{I}^{2}$ statistical parameter. Stratified analyses were conducted to assess modification effects by selected factors. Publication bias was examined by using Funnel plot and Egger's regression asymmetry test. Random-effects meta-analyses were conducted using the metafor package in $\mathrm{R}$

Results Of 260 identified articles, 25 were included in the present review. Comparing the highest vs. the lowest category of prenatal $25(\mathrm{OH}) \mathrm{D}$ levels, the pooled beta coefficients were $0.95(95 \%$ CI $-0.03,1.93 ; \mathrm{p}=0.05)$ for cognition, and 0.88 (95\% CI $-0.18,1.93 ; \mathrm{p}=0.10)$ for psychomotor development. The pooled relative risk for ADHD was 0.72 (95\% CI, 0.59, $0.89 ; \mathrm{p}=0.002)$, and the pooled odds ratio for autism-related traits was $0.42(95 \% \mathrm{CI}, 0.25,0.71 ; \mathrm{p}=0.001)$. Stratified analyses showed stronger associations between $25(\mathrm{OH}) \mathrm{D}$ concentrations in early-mid pregnancy and cognition (beta coefficient 1.18, 95\% CI -0.16, 2.51; p=0.08), psychomotor performance (beta coefficient 1.43, 95\% CI $-0.65,3.52$; $\mathrm{p}=0.18$ ), and risk of autism-related traits (OR 0.28, 95\% CI $0.19,0.42 ; \mathrm{p}<0.01)$. There was little evidence for protective effects of high prenatal $25(\mathrm{OH}) \mathrm{D}$ for language development and behavior difficulties.

Conclusion This meta-analysis provides supporting evidence that increased prenatal exposure to $25(\mathrm{OH}) \mathrm{D}$ levels is associated with improved cognitive development and reduced risk of ADHD and autism-related traits later in life. Associations represent a potentially high public health burden given the current prevalence of vitamin D deficiency and insufficiency among childbearing aging and pregnant women.

\section{OP77 METHODS TO INCREASE RESPONSE RATES TO A POPULATION-BASED MATERNITY SURVEY: A COMPARISON OF TWO PILOT STUDIES}

S Harrison, J Henderson, F Alderdice, MA Quigley*. National Perinatal Epidemiology Unit, University of Oxford, Oxford, UK

\subsection{6/jech-2019-SSMabstracts.80}

Background Surveys are established methods for collecting population data that are unavailable from other sources; however, response rates to surveys are declining. This decline is exemplified in the National Maternity Surveys which are carried out in England at regular intervals by the National Perinatal Epidemiology Unit; the response rate declined from 63\% in 2006 to $29 \%$ in 2016. A number of methods have been identified to increase survey returns yet response rates continue to fall. We evaluated the impact of five selected methods on the response rate to pilot surveys, conducted prior to the 2018 National Maternity Survey.

Methods The pilot National Maternity Surveys were cross-sectional population-based questionnaire surveys of women who were three months postpartum selected at random from birth registrations. Women received a postal questionnaire, which they could complete on paper, online or verbally over the telephone. An initial pilot survey was conducted (pilot 1, $\mathrm{n}=1,000$ ) to which the response rate was lower than expected. Therefore, a further pilot survey was conducted (pilot 2, $\mathrm{n}=2,000$ ) using additional selected methods with the specific aim of increasing the response rate. The additional selected methods used for all women in pilot 2 were: pre- notification, a shorter questionnaire, more personable survey materials, an additional reminder, and inclusion of quick response (QR) codes to enable faster access to the online version of the survey. To assess the impact of the selected methods, response rates to pilot surveys 1 and 2 were compared.

Results The response rate increased significantly from $28.7 \%$ in pilot 1 to $33.1 \%$ in pilot $2(+4.4 \%, 95 \% \mathrm{CI}: 0.88-7.83$, $\mathrm{p}=0.02$ ). Analysis of weekly returns according to time from initial and reminder mail-outs suggests that this increase was largely due to the additional reminder. Most respondents completed the paper questionnaire rather than taking part online or over the telephone in both pilot surveys. However, the overall response to the online questionnaire almost doubled from $1.8 \%$ in pilot 1 to $3.5 \%$ in pilot 2 , corresponding to an absolute difference of $1.7 \%$ (95\%CI:0.45-2.81, p=0.01), suggesting that $\mathrm{QR}$ codes might have facilitated online participation.

Conclusion Declining survey response rates may be ameliorated with the use of selected methods. Further studies should evaluate the effectiveness of each of these methods using randomised controlled trials and identify novel strategies for engaging populations in survey research.

\section{Methodological Issues}

\section{OP78 A CAUSAL INFERENCE PERSPECTIVE ON COMPOSITIONAL DATA AND COLLIDER 'BIAS'}

1,2 KF Arnold*, 1,2 L Berrie, 1,2,3PWG Tennant, 1,2,3 MS Gilthorpe. 'Leeds Institute for Data Analytics, University of Leeds, Leeds, UK; ${ }^{2}$ School of Medicine, University of Leeds, Leeds, UK; ${ }^{3}$ The Alan Turing Institute, London, UK

\subsection{6/jech-2019-SSMabstracts.81}

Background Compositional data (CD) comprise the parts of some whole, for which all parts sum to that whole; the whole may vary across individual units of analysis or remain fixed. Such data are common in many contexts, where interest often lies in understanding the effect of a particular part in relation to a subsequent outcome. Many of the inherent challenges associated with analysing CD have been discussed previously, though not within a formal causal framework by utilising directed acyclic graphs (DAGs). We use DAGs to consider the specific issue of collider bias as it pertains to CD.

Methods We demonstrate how to depict CD using DAGs, and identify two distinct effect estimands in the generic case: (1) the 'unbiased' (total) effect, and (2) the 'collider biased' effect. We consider each effect in the context of three specific example scenarios involving CD with variable or fixed totals: (1) the relationship between the economically active population and area-level gross domestic product (GDP) (variable total); (2) the relationship between fat consumption and body weight (variable total); and (3) the relationship between time spent sedentary and body weight (fixed total). For each scenario, we consider the distinct interpretation of each effect, and the resulting implications for related analyses.

Results In scenario (1), the 'unbiased' effect represents the average change in GDP that results from adding economically active individuals to the area whilst doing nothing to the population of economically inactive individuals, whereas the 'collider biased' effect represents the average change that results from swapping economically inactive individuals for economically active ones. In scenario (2), the 'unbiased' effect 